\title{
Poetry
}

\section{A Body Now, Perhaps Once Someone}

Pallavi Aneja, $\mathrm{MD}^{1}$

\section{Abstract}

\section{Description}

Personally driven by compassion in hospital medicine, I can't help but pen down certain emotions we experience as caregivers. It feels too selfish to not share some of those emotions, as profound as they are, though we seldom are aware that such feelings exist, even as they become palpable during adverse outcomes. Most of the time, thankfully the sick patients we encounter, recover and are discharged home. There are smiles of relief and gratitude to our profession as healers. But at times, with certain incurable conditions, we only can treat and try our best as doctors. There are tears, broken hearts, feelings of dread towards an approaching, certain end that we bitterly fight against, but lose. Commiserating with a patient in pain and with their loved ones is natural as a physician. And in this work, I share one of those unfortunate moments.

\section{Keywords}

poetry; medicine in the arts; frail elderly; widowhood; physician-patient relations; attitude to death; compassion

\section{A Body Now, Perhaps Once Someone}

Skin so pale, a bare lamination, to osseous matter, garbed in a hospital gown

Cadaverous, perhaps once someone

wearing a smile cemented

Stark now, a while ago perchance articulate

As ice was she, but not cold yet

For he stood beside her

Off the cuff, somber,

forever her buddy, paramour,

of decades now her husband

Breathing in torment

Warm was he, yet frozen.

A dead body of a frail old lady

Saw we all, but he

As his youth, love and happiness,

dreams and fulfilled until the past hour promises,

everything called life, to him was she

the person, beneath the skin so pale, a bare lamination, Transparent osseous matter garbed in a hospital gown cadaverous, perhaps once someone!

Author affiliations are listed at the end of this article.

Correspondence to: Pallavi Aneja, MD 401 NW 42nd Avenue Plantation, FL 33317

(Pallavi.Aneja@ hcahealthcare.com) 


\section{Conflicts of Interest}

The author declares she has no conflicts of interest.

Dr Aneja is an employee of Westside Regional Medical Center, a hospital affiliated with the journal's publisher.

This research was supported (in whole or in part) by HCA Healthcare and/or an

HCA Healthcare affiliated entity. The views expressed in this publication represent those of the author(s) and do not necessarily represent the official views of HCA Healthcare or any of its affiliated entities.

\section{Author Affiliation}

1. Westside Regional Medical Center, Plantation, $\mathrm{FL}$ 